\title{
Energy formulation for flexural - torsional buckling of thin-walled column with open cross- section
}

\author{
Charles Chinwuba Ike
}

Department of Civil Engineering, Enugu State University of Science and Technology, Enugu 400001, Nigeria

Corresponding Author Email: ikecc2007@yahoo.com

https://doi.org/10.18280/mmep.050202

Received: 7 April 2018

Accepted: 4 June 2018

\section{Keywords:}

flexural - torsional buckling, thin-walled columns with open cross-sections, energy formulation, euler - lagrange differential equation

\begin{abstract}
In this work, the problem of flexural - torsional buckling analysis of thin-walled column with open cross-section has been formulated using energy methods. Thin-walled column with open cross-section of arbitrary slope was considered. The deformation taking place during elastic buckling was assumed to consist of a combination of twisting and bending about two axes of the cross-section. The total potential energy functional was derived as the sum of the strain energy and the potential energy of the loads. Euler - Lagrange differential equations were used to obtain the differential equations corresponding to the conditions for the minimization of the total potential energy functional. It was found that the integral formulation reduced to a boundary value problem represented by a system of three coupled differential equations in terms of three unknowns which were the three displacement variables, two translational displacements $\mathrm{u}(\mathrm{z}), \mathrm{v}(\mathrm{z})$ and one rotational displacement $\theta(\mathrm{z})$.
\end{abstract}

\section{INTRODUCTION}

Thin-walled columns with open cross-sectional shapes such as $+, I, T, L$ and $[$ are frequently used in a variety of structures (such as buildings, bridges, aerospace, aeronautical and marine structures); and can be made with isotropic or anisotropic materials. The sections are called cruciform, $(+)$, tee $(T)$, angle $(\llcorner)$, cee $([)$ and I $(I)$. The sections can be singly-symmetric, doubly-symmetric or unsymmetric. Such thin-walled columns with open cross-sections have low torsional stiffness, and hence are prone to torsional buckling and instabilities [1-5].

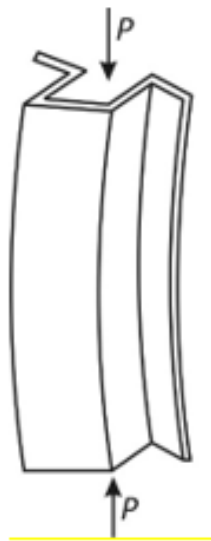

Figure 1. Flexural - torsional buckling mode of a $\longleftarrow$ shaped thin-walled column

Thin-walled columns with open cross-sections can fail under compressive loads by buckling in the following modes: flexural (Euler) buckling about one of the principal axes, torsional buckling about the shear centre, or a combination of both flexural and torsional buckling called flexural-torsional buckling shown in Figure 1. Flexural (Euler) buckling shown in Figure 2 is the primary type of buckling where the column becomes unstable due to deflection about the axis with smallest radius of gyration (i.e. largest slenderness ratio) [5].

Torsional buckling shown in Figure 3 involves twisting of the cross-section. In such cases, the elastic critical buckling stress depends partly on the Saint Venant torsional rigidity and on the warping rigidity [4]. Torsional buckling can only properly take place when the shear centre and the geometrical centroid of the cross-section coincide, i.e. in symmetrical cross-sections.

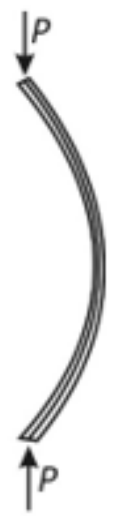

Figure 2. Flexural (Euler) buckling mode

The modes of buckling failure do not always occur independently, and interaction of the buckling modes can occur. Flexural and torsional displacements of the thin walled column take place simultaneously when the shear centre and the geometrical centroid of the cross-section are not coincident. This results in a decrease of the critical buckling load in the flexural - torsional buckling problem as compared to the flexural (Euler) buckling load [4, 5]. Flexural-torsional buckling consists in failure of the axially compressed thin 
walled column with open cross-section due to a combination of flexural buckling and torsional buckling as a result of simultaneous flexure (bending) and twisting of the crosssection $[6,7]$. This buckling mode is common in thin-walled columns with unsymmetrical cross-sections, including those with one-axis of symmetry such as channels, structural tees, double angles and those without any axis of symmetry and unequal legs single angle sections.

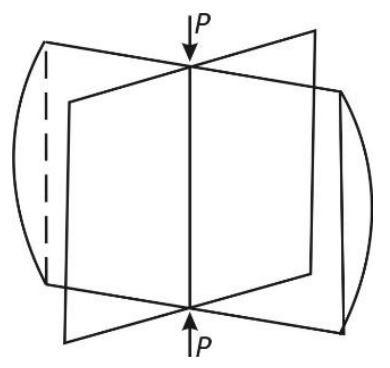

Figure 3. Torsional buckling mode of a + shaped thin-walled column

The flexural - torsional buckling problems of thin walled columns have been studied by Alsayad [8], Timoshenko and Gere [9], Chajes [10], Wang et al [11], and Athen and Bulson [12] and used in the formulation and development of design criteria for design in structural steel [13]. Ike et al [14] have used the Galerkin variational method to find the buckling loads and buckling modal shapes of thin walled columns with open cross-sections.

Mama et al [15] have used the finite Fourier sine transform method to solve the flexural -torsional buckling problem of thin-walled columns with open cross-sections. Onah et al [16] used the Fourier series method to solve the flexural-torsional problem of thin-walled columns with open cross-sections. Other researchers who have worked on torsion are Det [17] and Trahair [18].

The energy method was adopted in this study because it is a versatile mathematical and numerical tool for the formulation and solution of many problems in structures. It offers an alternative way of formulation of boundary value problems, by representing them as integral statements. It is also a basis for the approximate solutions of problems of elasticity and stability. It can also be used to obtain closed form analytical solutions to structural problems. The academic value of the present study is to derive from first principles considerations the governing equations for the linear elastic buckling problem of thin walled column with open cross-sections, and then to use a Ritz direct variational method to obtain expressions for the buckling loads and buckling moles.

\subsection{Research aim and objectives}

The general aim of this work is to use the energy method to formulate the problem of flexural - torsional buckling of thinwalled columns with open cross-sections. The specific objectives are:

(i) to determine the total potential energy functional $\Pi$ of a thin-walled column with open cross-section undergoing flexural-torsional buckling.

(ii) to use the Euler - Lagrange differential equations to find the conditions for the minimization of the total potential energy of the system. (iii) to obtain the differential equation for the flexuraltorsional buckling analysis of thin-walled columns with open cross-sections.

(iv) to obtain elastic buckling solutions using energy formulation for thin-walled columns with open cross-sections for the case of hinged ends $z=0$, and $z=l$ for (a) doubly-symmetric cross-sectional shapes, (b) singly-symmetric cross-sectional shapes, and (c) asymmetric cross-sections.

\section{THEORETICAL FRAMEWORK}

Assumptions of the formulation.

The energy formulation is based on the following assumptions:

(i) the thin-walled column considered undergoes flexural deformation about the two axes of the open cross-section. The shear centre of the open crosssection and the other points on the open cross-section undergo translational displacements denoted by $u$ and $v$, respectively, about the $x$ and $y$ axes of the crosssection.

(ii) the column considered undergoes twisting deformation. The open cross-section has a rotational displacement about the shear centre, which produces additional translational displacement components denoted by $u_{1}$ and $v_{1}$ respectively, about the $x$ and $y$ axes of the cross-section.

\section{METHODOLOGY}

\subsection{Total potential energy functional $\Pi$}

The total potential energy functional $\Pi$ for the thin-walled column with open cross-section under flexural-torsional buckling is the sum of the strain energy functional $U$ and the potential energy due to the external compressive load $V_{e}$.

$\Pi=U+V_{e}$

The shear centre of the cross-section is chosen as the origin. The $x$ and $y$ coordinate axes are assumed to be coincident with the principal axes of the open cross-section, and the $z$ coordinate axis is the longitudinal axis of the thin-walled column through the shear centre.

\subsection{Strain energy functional}

The strain energy functional $U$, for the column with open cross-section under flexural - torsional buckling is the sum of four component parts: the strain energy due to bending deformation in the $x$ and $y$ coordinate directions of the open cross-section of the column denoted by $U_{b x}$ and $U_{b y}$ respectively, the strain energy due to Saint Venant shear stress deformation denoted by $U_{s v}$, and the strain energy of the longitudinal stresses due to warping torsion denoted by $U_{w t}$.

For the column with open cross-section considered, the longitudinal axis is defined as the $z$ coordinates axis, and the open cross-section is on the $x y$ coordinate plane, the strain energy functional, $U$, is given by 
$U=U_{b y}+U_{b x}+U_{s v}+U_{w t}$

$U=\frac{1}{2} \int_{0}^{l} E I_{x x}\left(v^{\prime \prime}(z)\right)^{2} d z+\frac{1}{2} \int_{0}^{l} E I_{y y}\left(u^{\prime \prime}(z)\right)^{2} d z$

$+\frac{1}{2} \int_{0}^{l} G I_{t}\left(\theta^{\prime}(z)\right)^{2} d z+\frac{1}{2} \int_{0}^{l} E I_{w w}\left(\theta^{\prime \prime}(z)\right)^{2} d z$

where, $E$ is the Young's modulus of the column material

$I_{x x}$ is the principal moment of inertia about the $x$-axis

$I_{y y}$ is the principal moment of inertia about the $y$-axis

$G$ is the shear modulus or modulus of rigidity of the column material

$I_{t}$ is the St Venant torsion constant or the torsional moment of inertia

$I_{w w}$ is the principal warping constant or principal warping moment of inertia

$u(z)$ is the translational displacement in the $x$-direction

$v(z)$ is the translational displacement in the $y$ direction

$\theta(z)$ is the rotational displacement

and the primes represent differentiation with respect to the

space coordinate variable in the longitudinal axis of the member, $(z)$.

Equation (3) is simplified to:

$U=\frac{1}{2} \int_{0}^{l}\left\{\begin{array}{l}E I_{x x}\left(v^{\prime \prime}(z)\right)^{2}+E I_{y y}\left(u^{\prime \prime}(z)\right)^{2} \\ +G I_{t}\left(\theta^{\prime}(z)\right)^{2}+E I_{w w}\left(\theta^{\prime \prime}(z)\right)^{2}\end{array}\right\} d z$

\subsection{Potential energy due to the external axial compressive loads, $V_{e}$}

The potential energy expression due to the external loads is found from the consideration of the work done by the external loads as the thin-walled column deforms under the axial compression. This is given by the work integral over the crosssectional area of the thin-walled column given by the double integral:

$$
V_{e}=-\iint_{A} P \delta_{z}
$$

where $A$ the domain of the double integral, is the crosssectional area of the column.

$V_{e}=-\iint_{A} \sigma_{z z} d A \delta_{z}$

where $\sigma_{z z}=\frac{P}{A}$

$\delta_{z}$ is the differential between the arc length and the chord length of a longitudinal fibre of the thin-walled column after deformation due to the axial compressive load, $P$.

From a consideration of an arbitrary element $d z$ of the thinwalled column member, we can find the elemental length $d s$ of the deformed thin-walled column element using vector calculus/geometry as:

$|d r|=d s=\left(d z^{2}+d \bar{u}^{2}+d \bar{v}^{2}\right)^{1 / 2}$ where the $x$ and $y$ components of the displacements of the upper and lower ends of the thin-walled column element are $\bar{u}, \bar{v}$ and $\bar{u}+d \bar{u}, \bar{v}+d \bar{v}$

$d \vec{r}=d u i+d v j+d z k$

where $i, j, k$ are unit vectors of the three dimensional Cartesian coordinate system.

Applying the dot product,

$d s^{2}=|d r|^{2}=d r \cdot d r=d \bar{u}^{2}+d \bar{v}^{2}+d z^{2}$

or

$d s^{2}=d z^{2}+d \bar{u}^{2}+d \bar{v}^{2}$

$\frac{d s^{2}}{d z^{2}}=\left(1+\frac{d \bar{u}^{2}}{d z^{2}}+\frac{d \bar{v}^{2}}{d z^{2}}\right)$

$d s^{2}=\left(1+\frac{d \bar{u}^{2}}{d z^{2}}+\frac{d \bar{v}^{2}}{d z^{2}}\right) d z^{2}$

$d s=\left[1+\left(\frac{d \bar{u}}{d z}\right)^{2}+\left(\frac{d \bar{v}}{d z}\right)^{2}\right]^{1 / 2} d z$

$d s=\left[1+\frac{1}{2}\left(\frac{d \bar{u}}{d z}\right)^{2}+\frac{1}{2}\left(\frac{d \bar{v}}{d z}\right)^{2}\right] d z$

By integration,

$s=\int_{0}^{l} d s=\int_{0}^{l}\left[1+\frac{1}{2}\left(\frac{d \bar{u}}{d z}\right)^{2}+\frac{1}{2}\left(\frac{d \bar{v}}{d z}\right)^{2}\right] d z$
$s=\int_{0}^{l} d z+\int_{0}^{l}\left[\frac{1}{2}\left(\frac{d \bar{u}}{d z}\right)^{2}+\frac{1}{2}\left(\frac{d \bar{v}}{d z}\right)^{2}\right] d z$

$s=l+\int_{0}^{l}\left[\frac{1}{2}\left(\frac{d \bar{u}}{d z}\right)^{2}+\frac{1}{2}\left(\frac{d \bar{v}}{d z}\right)^{2}\right] d z$

$s=l+\frac{1}{2} \int_{0}^{l}\left[\left(\frac{d \bar{u}}{d z}\right)^{2}+\left(\frac{d \bar{v}}{d z}\right)^{2}\right] d z$

$\delta_{z}=s-l=\frac{1}{2} \int_{0}^{l}\left[\left(\frac{d \bar{u}}{d z}\right)^{2}+\left(\frac{d \bar{v}}{d z}\right)^{2}\right] d z$

The translational displacement components $\bar{u}$ and $\bar{v}$ of the differential longitudinal element is the sum of the translational displacement of the shear centre, $u$ and $v$ and the translational displacement components $u_{1}, v_{1}$ due to the rotation of the element about the shear centre.

$u_{1}=r \theta \sin \alpha$

$v_{1}=r \theta \cos \alpha$ 
$u_{1}=y_{0} \theta$

$v_{1}=x_{0} \theta$

where $r \sin \alpha=y_{0}$

$r \cos \alpha=x_{0}$

Hence,

$\bar{u}=u-u_{1}=u-y_{0} \theta$

$\bar{v}=v+v_{1}=v+x_{0} \theta$

Thus,

$\delta_{z}=\frac{1}{2} \int_{0}^{l}\left\{\left[\frac{d}{d z}\left(u-y_{0} \theta\right)\right]^{2}+\left[\frac{d}{d z}\left(v+x_{0} \theta\right)\right]^{2}\right\} d z$

$\delta_{z}=\frac{1}{2} \int_{0}^{l}\left\{\left(\frac{d u}{d z}-y_{0} \frac{d \theta}{d z}\right)^{2}+\left(\frac{d v}{d z}+x_{0} \frac{d \theta}{d z}\right)^{2}\right\} d z$

$\delta_{z}=\frac{1}{2} \int_{0}^{l}\left\{\begin{array}{l}\left(\frac{d u}{d z}\right)^{2}-2 y_{0} \frac{d u}{d z} \frac{d \theta}{d z}+y_{0}^{2}\left(\frac{d \theta}{d z}\right)^{2} \\ +\left(\frac{d v}{d z}\right)^{2}+2 x_{0} \frac{d v}{d z} \frac{d \theta}{d z}+x_{0}^{2}\left(\frac{d \theta}{d z}\right)^{2}\end{array}\right\} d z$

$\delta_{z}=\frac{1}{2} \int_{0}^{l}\left\{\begin{array}{l}\left(\frac{d u}{d z}\right)^{2}+\left(\frac{d v}{d z}\right)^{2}+\left(x_{0}^{2}+y_{0}^{2}\right)\left(\frac{d \theta}{d z}\right)^{2} \\ +2 x_{0} \frac{d v}{d z} \frac{d \theta}{d z}-2 y_{0}\left(\frac{d u}{d z}\right)\left(\frac{d \theta}{d z}\right)\end{array}\right\} d z$

Then,

$V_{e}=-\iint_{A} \sigma_{x x} d A \delta_{z}$

$V_{e}=-\frac{1}{2} \iint_{0}^{l} \int_{A} \sigma_{x x}\left[\begin{array}{l}\left(\frac{d u}{d z}\right)^{2}+\left(\frac{d v}{d z}\right)^{2}+\left(x_{0}^{2}+y_{0}^{2}\right)\left(\frac{d \theta}{d z}\right)^{2} \\ +2 x_{0}\left(\frac{d v}{d z}\right)\left(\frac{d \theta}{d z}\right)-2 y_{0}\left(\frac{d u}{d z}\right)\left(\frac{d \theta}{d z}\right)\end{array}\right] d A d z$

We employ the geometrical properties of cross-sections, thus:

$\iint_{A} d A=A$

$\iint_{A} y_{0} d A=y_{0} A$

$\iint_{A} x_{0} d A=x_{0} A$

$\iint_{A}\left(x_{0}^{2}+y_{0}^{2}\right) d A=i_{0}^{2} A$ where $x_{0}$ and $y_{0}$ are the centroidal coordinates of the open cross-section of the thin-walled column, $i_{0}$ is the polar radius of gyration of the thin-walled column cross-section with respect to the shear centre.

$$
\begin{aligned}
& V_{e}=-\frac{1}{2}\left\{\int_{0}^{l}\left[\left(\frac{d u(z)}{d z}\right)^{2}+\left(\frac{d v(z)}{d z}\right)^{2}\right] d z \iint_{A} \frac{P}{A} d A\right. \\
& +\iint_{A}\left(x_{0}^{2}+y_{0}^{2}\right) \frac{P}{A} d A \int_{0}^{l}\left(\frac{d \theta(z)}{d z}\right)^{2} d z+ \\
& \left.\int_{0}^{l} \frac{d v(z)}{d z} \frac{d \theta(z)}{d z} d z \iint_{A} 2 x_{0} \frac{P}{A} d A-\int_{0}^{l} \frac{d u(z)}{d z} \frac{d \theta(z)}{d z} d z \iint_{A} 2 y_{0} \frac{P}{A} d A\right\}
\end{aligned}
$$

$$
\begin{aligned}
& V_{e}=-\frac{1}{2}\left\{P \int_{0}^{l}\left[\left(\frac{d u(z)}{d z}\right)^{2}+\left(\frac{d v(z)}{d z}\right)^{2}\right] d z\right. \\
& +P \cdot i_{0}^{2} \int_{0}^{l}\left(\frac{d \theta(z)}{d z}\right)^{2} d z+2 P x_{0} \int_{0}^{l} \frac{d v(z)}{d z} \frac{d \theta(z)}{d z} d z- \\
& \left.2 P y_{0} \int_{0}^{l} \frac{d u(z)}{d z} \frac{d \theta(z)}{d z} d z\right\} \\
& V_{e}=\frac{-P}{2} \int_{0}^{l}\left[\left(\frac{d u(z)}{d z}\right)^{2}+\left(\frac{d v(z)}{d z}\right)^{2}+i_{0}^{2}\left(\frac{d \theta(z)}{d z}\right)^{2}\right. \\
& \left.-2 y_{0}\left(\frac{d u(z)}{d z}\right)\left(\frac{d \theta(z)}{d z}\right)+2 x_{0}\left(\frac{d v(z)}{d z}\right)\left(\frac{d \theta(z)}{d z}\right)\right] d z \\
& V_{e}=\frac{-P}{2} \int_{0}^{l}\left[\begin{array}{l}
\left(u^{\prime}(z)\right)^{2}+\left(v^{\prime}(z)\right)^{2}+i_{0}^{2}\left(\theta^{\prime}(z)\right)^{2}- \\
2 y_{0} u^{\prime}(z) \theta^{\prime}(z)+2 x_{0} v^{\prime}(z) \theta^{\prime}(z)
\end{array}\right] d z \\
& \Pi=\frac{1}{2} \int_{0}^{l}\left[\begin{array}{l}
E I_{x x}\left(v^{\prime \prime}(z)^{2}+E I_{y y}\left(u^{\prime \prime}(z)\right)^{2}\right. \\
+G I_{t}\left(\theta^{\prime}(z)\right)^{2}+E I_{w w}\left(\theta^{\prime \prime}(z)\right)^{2}
\end{array}\right] d z- \\
& \frac{P}{2} \int_{0}^{l}\left[\begin{array}{c}
\left(u^{\prime}(z)\right)^{2}+\left(v^{\prime}(z)\right)^{2}+i_{0}^{2}\left(\theta^{\prime}(z)\right)^{2} \\
+2 x_{0} v^{\prime}(z) \theta^{\prime}(z)-2 y_{0} u^{\prime}(z) \theta^{\prime}(z)
\end{array}\right] d z \\
& \Pi=\frac{1}{2} \int_{0}^{l}\left\{\begin{array}{l}
E I_{x x}\left(v^{\prime \prime}(z)^{2}\right)+E I_{y y}\left(u^{\prime \prime}(z)\right)^{2} \\
+G I_{t}\left(\theta^{\prime}(z)\right)^{2}+E I_{w w}\left(\theta^{\prime \prime}(z)\right)^{2}-
\end{array}\right. \\
& \left.P\left[\begin{array}{c}
\left(\left(u^{\prime}(z)\right)^{2}+\left(v^{\prime}(z)\right)^{2}+i_{0}^{2}\left(\theta^{\prime}(z)\right)^{2}\right. \\
+2 x_{0} v^{\prime}(z) \theta^{\prime}(z)-2 y_{0} u^{\prime}(z) \theta^{\prime}(z)
\end{array}\right]\right\} d z
\end{aligned}
$$

\section{RESULTS}

The total energy functional is of the general integral form:

$$
\Pi=\frac{1}{2} \int_{0}^{l} F\left(u^{\prime}(z), v^{\prime}(z), \theta^{\prime}(z), u^{\prime \prime}(z), v^{\prime \prime}(z), \theta^{\prime \prime}(z)\right) d z
$$

where the integrand is given by: 


$$
\begin{aligned}
& F=E I_{y y} u^{\prime \prime}(z)+E I_{x x} v^{\prime \prime}(z)+E I_{w w} \theta^{\prime \prime}(z) \\
& +G I_{t}\left(\theta^{\prime}(z)\right)^{2}-P\left[\left(u^{\prime}(z)\right)^{2}+\left(v^{\prime}(z)\right)^{2}+\right. \\
& \left.i_{0}^{2}\left(\theta^{\prime}(z)\right)^{2}+2 x_{0} v^{\prime}(z) \theta^{\prime}(z)-2 y_{0} u^{\prime}(z) \theta^{\prime}(z)\right]
\end{aligned}
$$

From the principle of minimization of the total potential energy functional, the condition for extremization (minimization) of the total potential energy functional given generally by Equation (45) is obtained from the Euler Lagrange equations.

For the first variation of the total potential energy $\Pi$ to vanish,

$$
\partial \Pi=0
$$

We obtain the system of Euler - Lagrange differential equations:

$$
\begin{aligned}
& -\frac{d}{d z}\left(\frac{\partial F}{\partial u^{\prime}(z)}\right)+\frac{d^{2}}{d z^{2}}\left(\frac{\partial F}{\partial u^{\prime \prime}(z)}\right)=0 \\
& -\frac{d}{d z}\left(\frac{\partial F}{\partial v^{\prime}(z)}\right)+\frac{d^{2}}{d z^{2}}\left(\frac{\partial F}{\partial v^{\prime \prime}(z)}\right)=0 \\
& -\frac{d}{d z}\left(\frac{\partial F}{\partial \theta^{\prime}(z)}\right)+\frac{d^{2}}{d z^{2}}\left(\frac{\partial F}{\partial \theta^{\prime \prime}(z)}\right)=0
\end{aligned}
$$

From Equation (46), we obtain by differentiation:

$$
\begin{aligned}
& \frac{\partial F}{\partial u^{\prime}(z)}=-P\left(2 u^{\prime}(z)-2 y_{0} \theta^{\prime}(z)\right) \\
& \frac{\partial F}{\partial u^{\prime \prime}(z)}=E I_{y y} 2 u^{\prime \prime}(z)
\end{aligned}
$$

$\frac{\partial F}{\partial v^{\prime}(z)}=-P\left(2 v^{\prime}(z)+2 x_{0} \theta^{\prime}(z)\right)$

$$
\frac{\partial F}{\partial v^{\prime \prime}(z)}=E I_{x x} 2 v^{\prime \prime}(z)
$$

$\frac{\partial F}{\partial \theta^{\prime}(z)}=2 G I_{t} \theta^{\prime}(z)-P\left(2 i_{0}^{2} \theta^{\prime}(z)+2 x_{0} v^{\prime}(z)-2 y_{0} u^{\prime}(z)\right)$

$\frac{\partial F}{\partial \theta^{\prime \prime}(z)}=2 E I_{w w} \theta^{\prime \prime}(z)$

Thus, the Euler - Lagrange equations of flexural-torsional buckling problem of thin-walled columns with open crosssections become:

$$
\begin{aligned}
& \frac{-d}{d z}\left(-P\left(2 u^{\prime}(z)-2 y_{0} \theta^{\prime}(z)\right)+\frac{d^{2}}{d z^{2}} E I_{y y} 2 u^{\prime \prime}(z)=0\right. \\
& \frac{d^{2}}{d z^{2}} E I_{y y} u^{\prime \prime}(z)+P\left(u^{\prime \prime}(z)-y_{0} \theta^{\prime \prime}(z)\right)=0
\end{aligned}
$$

$$
\frac{-d}{d z}\left(-P\left(2 v^{\prime}(z)+2 x_{0} \theta^{\prime}(z)\right)+\frac{d^{2}}{d z^{2}}\left(E I_{x x} 2 v^{\prime \prime}(z)\right)=0\right.
$$

$\frac{d^{2}}{d z^{2}} E I_{x x} v^{\prime \prime}(z)+P\left(v^{\prime \prime}(z)+x_{0} \theta^{\prime \prime}(z)\right)=0$

$\frac{-d}{d z}\left(2 G I_{t} \theta^{\prime}(z)-P\left(2 i_{0}^{2} \theta^{\prime}(z)+2 x_{0} v^{\prime}(z)-2 y_{0} u^{\prime}(z)\right)\right)$

$+\frac{d^{2}}{d z^{2}} 2 E I_{w w} \theta^{\prime \prime}(z)=0$

$\frac{d^{2}}{d z^{2}} E I_{w w} \theta^{\prime \prime}(z)-\frac{d}{d z} G I_{t} \theta^{\prime}(z)$

$+P\left(i_{0}^{2} \theta^{\prime \prime}(z)+x_{0} v^{\prime \prime}(z)-y_{0} u^{\prime \prime}(z)\right)=0$

For prismatic cross-sections, and homogeneous thin-walled column materials we obtain the system of coupled differential equations:

$$
\begin{aligned}
& E I_{y y} u^{i v}(z)+P\left(u^{\prime \prime}(z)-y_{0} \theta^{\prime \prime}(z)\right)=0 \\
& E I_{x x} v^{i v}(z)+P\left(v^{\prime \prime}(z)+x_{0} \theta^{\prime \prime}(z)\right)=0 \\
& E I_{w w} \theta^{i v}(z)-G I_{t} \theta^{\prime \prime}(z)+P\left(i_{0}^{2} \theta^{\prime \prime}(z)+x_{0} v^{\prime \prime}(z)-y_{0} u^{\prime \prime}(z)\right)=0
\end{aligned}
$$

The system of coupled differential equations in terms of the unknown displacement functions $u(z), v(z)$ and $\theta(z)$ are called the Timoshenko equations for the flexural lateral - torsional buckling of thin walled columns.

\subsection{Elastic buckling solutions for simply supported thin- walled columns}

For thin-walled columns simply supported at the ends $z=0$ and $z=l$, the translational displacements in the $x$ and $y$ coordinate directions, and the bending moment about these axes vanish. The boundary conditions are

$$
\begin{aligned}
& u(z=0)=0 \\
& u(z=l)=0 \\
& v(z=0)=0 \\
& v(z=l)=0
\end{aligned}
$$

$\frac{d^{2} u}{d z^{2}}(z=0)=u^{\prime \prime}(z=0)=0$

$\frac{d^{2} u}{d z^{2}}(z=l)=u^{\prime \prime}(z=l)=0$

$\frac{d^{2} v}{d z^{2}}(z=0)=v^{\prime \prime}(z=0)=0$ 
$\frac{d^{2} v}{d z^{2}}(z=l)=v^{\prime \prime}(z=l)=0$

The boundary conditions corresponding to the zero rotation and zero warping restraint at the hinged ends $z=0$, and $z=l$ are:

$\theta(z=0)=0$

$\theta(z=l)=0$

$\frac{d^{2} \theta}{d z^{2}}(z=0)=\theta^{\prime \prime}(z=0)=0$

$\frac{d^{2} \theta}{d z^{2}}(z=l)=\theta^{\prime \prime}(z=l)=0$

A suitable buckling shape function that satisfies the boundary conditions is the sinusoidal function. The buckling modal functions are thus:

$u(z)=c_{1 m} \sin \frac{m \pi z}{l}$

$v(z)=c_{2 m} \sin \frac{m \pi z}{l}$

$\theta(z)=c_{3 m} \sin \frac{m \pi z}{l}$

where $c_{1 m}, c_{2 m}$ and $c_{3 m}$ are the undetermined parameters of the buckling modal functions.

The total strain energy functional is thus from Equation (3),

$U=\frac{1}{2} \int_{0}^{l} E I_{y y} c_{1 m}^{2}\left(\frac{m \pi}{l}\right)^{4} \sin ^{2} \frac{m \pi z}{l} d z+\frac{1}{2} \int_{0}^{l} E I_{x x} c_{2 m}^{2}\left(\frac{m \pi}{l}\right)^{4} \sin ^{2} \frac{m \pi z}{l} d z+$

$\frac{1}{2} \int_{0}^{l} G I_{t} c_{3 m}^{2}\left(\frac{m \pi}{l}\right)^{2} \cos ^{2} \frac{m \pi z}{l} d z+\frac{1}{2} \int_{0}^{l} E I_{w w} c_{3 m}^{2}\left(\frac{m \pi}{l}\right)^{4} \sin \frac{m \pi z}{l} d z(81)$

$U=\frac{1}{2} E I_{y y} c_{1 m}^{2}\left(\frac{m \pi}{l}\right)^{4} \int_{0}^{l} \sin ^{2} \frac{m \pi z}{l} d z+\frac{1}{2} E I_{x x} c_{2 m}^{2}\left(\frac{m \pi}{l}\right)^{4} \int_{0}^{l} \sin ^{2} \frac{m \pi z}{l} d z+$

$\frac{1}{2} G I_{t} c_{3 m}^{2}\left(\frac{m \pi}{l}\right)^{2} \int_{0}^{l} \cos ^{2} \frac{m \pi z}{l} d z+\frac{1}{2} E I_{w w} c_{3 m}^{2}\left(\frac{m \pi}{l}\right)^{4} \int_{0}^{l} \sin ^{2} \frac{m \pi z}{l} d z$

Simplifying,

$U=\frac{1}{2} E I_{y y} c_{1 m}^{2}\left(\frac{m \pi}{l}\right)^{4} \frac{l}{2}+\frac{1}{2} E I_{x x} c_{2 m}^{2}\left(\frac{m \pi}{l}\right)^{4} \frac{l}{2}$

$+\frac{1}{2} G I_{t} c_{3 m}^{2}\left(\frac{m \pi}{l}\right)^{2} \frac{l}{2}+\frac{1}{2} E I_{w w} c_{3 m}^{2}\left(\frac{m \pi}{l}\right)^{4} \frac{l}{2}$

$U=\frac{1}{4} \frac{(m \pi)^{2}}{l}\left[\begin{array}{l}c_{1 m}^{2} E I_{y y}\left(\frac{m \pi}{l}\right)^{2}+c_{2 m}^{2} E I_{x x}\left(\frac{m \pi}{l}\right)^{2} \\ +c_{3 m}^{2}\left(G I_{t}+E I_{w w}\left(\frac{m \pi}{l}\right)^{2}\right)\end{array}\right]$
The potential energy due to the axial compressive force $P$ is:

$$
\begin{aligned}
& V_{e}=-\int_{0}^{l} \sigma_{z z} d A \delta_{z}=-\frac{1}{2} P \int_{0}^{l}\left\{\begin{array}{l}
c_{1 m}^{2}\left(\frac{m \pi}{l}\right)^{2} \cos ^{2} \frac{m \pi z}{l} \\
+c_{2 m}^{2}\left(\frac{m \pi}{l}\right)^{2} \cos ^{2}\left(\frac{m \pi z}{l}\right)+
\end{array}\right. \\
& \left(x_{0}^{2}+y_{0}^{2}\right) c_{3 m}^{2}\left(\frac{m \pi}{l}\right)^{2} \cos ^{2} \frac{m \pi z}{l} \\
& +2 x_{0} c_{2 m}\left(\frac{m \pi}{l}\right) \cos \frac{m \pi z}{l} c_{3 m}\left(\frac{m \pi}{l}\right) \cos \frac{m \pi z}{l}- \\
& \left.2 y_{0} c_{1 m}\left(\frac{m \pi}{l}\right) \cos \frac{m \pi z}{l} c_{3 m}\left(\frac{m \pi}{l}\right) \cos \frac{m \pi z}{l}\right\} d z
\end{aligned}
$$

$$
\begin{aligned}
V_{e}= & \frac{-P}{2}\left\{\begin{array}{l}
c_{1 m}^{2}\left(\frac{m \pi}{l}\right)^{2}+c_{2 m}^{2}\left(\frac{m \pi}{l}\right)^{2}+\left(x_{0}^{2}+y_{0}^{2}\right) c_{3 m}^{2}\left(\frac{m \pi}{l}\right)^{2} \\
+2 x_{0} c_{2 m} c_{3 m}\left(\frac{m \pi}{l}\right)^{2} \\
\end{array} \quad-2 y_{0} c_{1 m} c_{3 m}\left(\frac{m \pi}{l}\right)^{2} \int_{0}^{l} \cos ^{2} \frac{m \pi z}{l} d z\right.
\end{aligned}
$$

$$
V_{e}=\frac{-P}{4} \frac{(m \pi)^{2}}{l}\left\{c_{1 m}^{2}+c_{2 m}^{2}+c_{3 m}^{2} i_{0}-2 c_{1 m} c_{3 m} y_{0}+2 c_{2 m} c_{3 m} x_{0}\right\}
$$

The total potential energy functional of the thin-walled column is:

$$
\begin{aligned}
& \Pi=\frac{(m \pi)^{2}}{4 l}\left\{c_{1 m}^{2}\left(\left(\frac{m \pi}{l}\right)^{2} E I_{y y}-P\right)+c_{2 m}^{2}\left(\left(\frac{m \pi}{l}\right)^{2} E I_{x x}-P\right)+\right. \\
& \left.c_{3 m}^{2} i_{0}^{2}\left\{\frac{1}{i_{0}^{2}}\left(G I_{t}+E I_{w w}\left(\frac{m \pi}{l}\right)^{2}\right)-P\right\}+2 c_{1 m} c_{3 m} P y_{0}-2 c_{2 m} c_{3 m} P x_{0}\right\}
\end{aligned}
$$

But

$$
\begin{gathered}
P_{E_{y y}}=\left(\frac{m \pi}{l}\right)^{2} E I_{y y} \\
P_{E_{x x}}=\left(\frac{m \pi}{l}\right)^{2} E I_{x x}
\end{gathered}
$$

and $P_{\theta}=\frac{1}{i_{0}^{2}}\left(G I_{t}+\left(\frac{m \pi}{l}\right)^{2} E I_{w w}\right)$

where $P_{E_{y y}}$ is the Euler (flexural) buckling load in the $y y$ axis $P_{E_{x x}}$ is the Euler (flexural) buckling load in the $x x$ direction and $P_{\theta}$ in the torsional buckling load.

Then,

$\Pi=\frac{(m \pi)^{2}}{4 l}\left\{\begin{array}{l}c_{1 m}^{2}\left(P_{E_{y y}}-P\right)+c_{2 m}^{2}\left(P_{E_{x x}}-P\right)+i_{0}^{2} c_{3 m}^{2}\left(P_{\theta}-P\right) \\ +2 c_{1 m} c_{3 m} P y_{0}-2 c_{2 m} c_{3 m} P x_{0}\end{array}\right\}$ 
It is observed that the total potential energy functional in this case depends upon three sets of unknown generalised modal displacement parameters $c_{1 m}, c_{2 m}$ and $c_{3 m}$, where $m=1$, $2,3, \ldots$.

The total potential energy functional will assume a minimum or stationary value when it is made to vanish with respect to each of these three sets of unknown modal displacement parameters. Thus for,

$\delta \Pi=0$

We require three sets of equations:

$\frac{\partial \Pi}{\partial c_{1 m}}=0$

$\frac{\partial \Pi}{\partial c_{2 m}}=0$

$\frac{\partial \Pi}{\partial c_{3 m}}=0$

Thus,

$\frac{\partial \Pi}{\partial c_{1 m}}=c_{1 m}\left(P_{E_{y y}}-P\right)+c_{3 m} P y_{0}=0$

$\frac{\partial \Pi}{\partial c_{2 m}}=c_{2 m}\left(P_{E_{x x}}-P\right)-c_{3 m} P x_{0}=0$

$\frac{\partial \Pi}{\partial c_{3 m}}=c_{1 m} P y_{0}-c_{2 m} P x_{0}+c_{3 m} i_{0}^{2}\left(P_{\theta}-P\right)=0$

In matrix form, we obtain a system of three homogeneous equations in $c_{1 m}, c_{2 m}$ and $c_{3 m}$ as follows:

$$
\left(\begin{array}{ccc}
\left(P_{E_{y y}}-P\right) & 0 & P y_{0} \\
0 & \left(P_{E_{x x}}-P\right) & -P x_{0} \\
P y_{0} & -P x_{0} & i_{0}^{2}\left(P_{\theta}-P\right)
\end{array}\right)\left(\begin{array}{l}
c_{1 m} \\
c_{2 m} \\
c_{3 m}
\end{array}\right)=\left(\begin{array}{l}
0 \\
0 \\
0
\end{array}\right)
$$

The represents an eigenvalue eigenvector problem. For nontrivial solutions, the characteristic buckling equation is given by making the determinant of the coefficient matrix to vanish. Thus,

$$
\left|\begin{array}{ccc}
\left(P_{E_{y y}}-P\right) & 0 & P y_{0} \\
0 & \left(P_{E_{x x}}-P\right) & -P x_{0} \\
P y_{0} & -P x_{0} & i_{0}^{2}\left(P_{\theta}-P\right)
\end{array}\right|=0
$$

Expanding, we have;

$\left(P_{E_{y y}}-P\right)\left|\begin{array}{cc}\left(P_{E_{x x}}-P\right) & -P x_{0} \\ -P x_{0} & i_{0}^{2}\left(P_{\theta}-P\right)\end{array}\right|+P y\left|\begin{array}{cc}0 & \left(P_{E_{x x}}-P\right) \\ P y_{0} & -P x_{0}\end{array}\right|=0$
$\left(P_{E_{y y}}-P\right)\left(P_{E_{x x}}-P\right)\left(P_{\theta}-P\right)-\left(P_{E_{y y}}-P\right) \frac{P^{2} x_{0}^{2}}{i_{0}^{2}}$

$-\left(P_{E_{x x}}-P\right) \frac{P^{2} y_{0}^{2}}{i_{0}^{2}}=0$

The critical buckling load is found as the lowest buckling load which occurs when $m=1$, and

$P_{E_{y y}}=\frac{\pi^{2} E I_{y y}}{l^{2}}=P_{y y_{c r}}$

$P_{E_{x x}}=\frac{\pi^{2} E I_{x x}}{l^{2}}=P_{x x_{c}}$

$P_{\theta}=\frac{1}{i_{0}^{2}}\left(G I_{t}+\frac{\pi^{2} E I_{w w}}{l^{2}}\right)=P_{\theta_{c r}}$

where $P_{y y_{c r}}, P_{x x_{c r}}$ are the critical flexural buckling loads, and $P_{\theta_{c r}}$ is the critical torsional buckling load.

So, for the critical buckling load,

$\left(P_{y y_{c r}}-P\right)\left(P_{x x_{c r}}-P\right)\left(P_{\theta_{c r}}-P\right)-\left(P_{y y_{c r}}-P\right) \frac{P^{2} x_{0}^{2}}{i_{0}^{2}}$

$-\left(P_{x x_{c r}}-P\right) \frac{P^{2} y_{0}^{2}}{i_{0}^{2}}=0$

\subsection{Case of thin-walled column with doubly-symmetric open cross-section}

For thin-walled columns with doubly-symmetric open cross-sections, the geometrical centre coincides with the shear centre, and $x_{0}=0, y_{0}=0$.

The critical buckling equation simplifies to become:

$\left(P_{y y_{c r}}-P\right)\left(P_{x x_{c r}}-P\right)\left(P_{\theta_{c r}}-P\right)=0$

The three roots are the three critical buckling loads, given by:

$P=P_{x x_{c r}}=\frac{\pi^{2} E I_{x x}}{l^{2}}$

$P=P_{y y_{c r}}=\frac{\pi^{2} E I_{y y}}{l^{2}}$

$P=P_{\theta_{c r}}=\frac{1}{i_{0}^{2}}\left(G I_{t}+\frac{\pi^{2} E I_{w w}}{l^{2}}\right)$

The lowest value of $P$ would govern the buckling behaviour of the thin-walled column. 


\subsection{Case of thin walled column with singly-symmetric open cross-section}

For thin-walled columns with singly-symmetric open crosssections, one of the centroidal coordinates will be zero since the shear centre will lie on one of the axes of the cross-section. When the $y$ axis is the axis of symmetry, the shear centre will lie on the $y$ axis, and $x_{0}=0$, and the buckling equations become:

$$
\left(P_{y y_{c r}}-P\right)\left(P_{x x_{c r}}-P\right)\left(P_{\theta_{c r}}-P\right)-\left(P_{x x_{c r}}-P\right) \frac{P^{2} y_{0}^{2}}{i_{0}^{2}}=0
$$

This yields:

$$
\left(P_{x x_{c r}}-P\right)=0
$$

or, $P=P_{x x_{c r}}=\frac{\pi^{2} E I_{x x}}{l^{2}}$

and $\left(P_{y y_{c r}}-P\right)\left(P_{\theta_{c r}}-P\right)-\frac{P^{2} y_{0}^{2}}{i_{0}^{2}}=0$

Equation (116) is a quadratic equation in $P$. It has two buckling values corresponding to the two roots (zeros) of the quadratic equation. The equation represents the flexuraltorsional buckling equation. When the $x$ axis is the only axis of symmetry, the shear centre will be found on the $x$ axis, $y_{0}=$ 0 , and the characteristic buckling equation becomes:

$$
\begin{aligned}
& \left(P_{y y_{c r}}-P\right)\left(P_{x x_{c r}}-P\right)\left(P_{\theta_{c r}}-P\right)-\left(P_{y y_{c r}}-P\right) \frac{P^{2} x_{0}^{2}}{i_{0}^{2}}=0 \\
& \left(P_{y y_{c r}}-P\right)\left\{\left(P_{x x_{c r}}-P\right)\left(P_{\theta_{c r}}-P\right)-\frac{P^{2} x_{0}^{2}}{i_{0}^{2}}\right\}=0
\end{aligned}
$$

The buckling equations become:

$$
\begin{aligned}
& P=P_{y y_{c r}}=\frac{\pi^{2} E I_{y y}}{l^{2}} \\
& \left(P_{x x_{c r}}-P\right)\left(P_{\theta_{c r}}-P\right)-\frac{P^{2} x_{0}^{2}}{i_{0}^{2}}=0
\end{aligned}
$$

The governing flexural-torsional buckling load is the smaller root of the flexural-torsional buckling equation, and is obtained as:

$$
P_{F T}=\frac{1}{2 \alpha}\left\{P_{\theta_{c r}}+P_{x x_{c r}}-\sqrt{\left(P_{\theta_{c r}}+P_{x x_{c r}}\right)^{2}-4 \alpha P_{\theta_{c r}} P_{x x_{c r}}}\right\}
$$

where $\alpha=1-\left(\frac{x_{0}}{i_{0}}\right)^{2}$

If the $x$ axis is the only axis of symmetry, and

$$
P_{F T}=\frac{1}{2 \beta}\left\{P_{\theta_{c r}}+P_{y y_{c r}}-\sqrt{\left(P_{\theta_{c r}}+P_{y y_{c r}}\right)^{2}-4 \beta P_{\theta_{c r}} P_{y y_{c r}}}\right\}
$$

where $\beta=1-\left(\frac{y_{0}}{i_{0}^{2}}\right)^{2}$

if the $y$ axis is the only axis of symmetry.

\section{DISCUSSION}

The energy method has been successfully used in this study to derive the governing differential equations for the flexural - torsional buckling problem of thin-walled columns with open cross-sections. The total potential energy functional $\Pi$ for the problem was derived as Equation (44) from fundamental considerations as the sum of the strain energy of bending deformation about the $x$ and $y$ axes of the open crosssection, the strain energy of St Venant torsion, the strain energy of warping torsion and the potential energy of the applied compressive load. Euler - Lagrange differential equations, which are equivalent statements of the conditions for the minimization of the total potential energy functional were used to find the governing differential equations of flexural - torsional buckling of thin-walled columns with open cross-sections as Equations $(60-62)$ for the general case of non prismatic cross-sections and non homogeneous materials. For the case of prismatic cross-sections and homogeneous materials, the governing differential equations were found as Equations $(63-65)$.

The governing equations were found to be a system of three coupled differential equations in terms of three unknown displacement functions - two translational displacements $u(z)$, $v(z)$ and a rotational displacement $\theta(z)$.

Elastic buckling solutions for thin-walled columns with open cross-sectional shapes were obtained using the energy formulation for the case of hinged (simply supported) ends $z=$ 0 and $z=l$. The buckling modal functions where chosen to apriori satisfy the boundary conditions as Equations $(78-80)$ which were functions of three sets of unknown generalized modal displacement parameters. The total potential energy functional was evaluated as Equation (88) and found to depend on the three sets of unknown generalized displacement parameters. The condition of minimization of the potential energy functional was used to generate a set of three buckling equations, namely Equations (98 - 100). The buckling equations were expressed in matrix form and found to represent an algebraic eigenvalue eigenvector problem. The characteristic buckling equation was obtained as Equation (102) from the requirement of vanishing of the coefficient matrix in the homogeneous eigenvalue problem. Expansion of the characteristic buckling equation yielded the characteristic buckling equation as Equation (104). The equation for the critical buckling load was found in general as Equation (108). The critical buckling equation was found to simplify to Equation (109) for cases of thin-walled columns with doublysymmetric open cross-sections. The buckling modes are uncoupled for doubly-symmetrical sections, and the buckling equations are similarly uncoupled. The buckling loads were found for doubly-symmetric sections as Equations (110 112); with the lowest buckling load governing the behaviour.

For singly-symmetric open cross-sections, the buckling equations were found to simplify to Equation (113) for the case of the $y$ axis as the only axis of symmetry and to Equation (117) if the $x$ axis is the only axis of symmetry. The buckling equations in this case become Equations (115) and (116) for 
open cross-sections with $y$ axis as the only axis of symmetry and Equations (119) and (120) for open cross-sections with $x$ axis as the only axis of symmetry.

\section{CONCLUSION}

The conclusions of the study are as follows:

(i) The energy method can be used to formulate the flexural - torsional buckling problem of thin-walled columns with open cross-sections in variational form as a functional to be minimized.

(ii) The total potential energy functional for the flexural - torsional buckling problem of thin-walled columns with open cross-sections is a function of three unknown displacement functions and their derivatives, with respect to the column longitudinal $z$ coordinate variable (namely $u(z), v(z), \theta(z), u^{\prime \prime}(z)$, $v^{\prime \prime}(z)$ and $\left.\theta^{\prime \prime}(z)\right)$.

(iii) The differential equations governing the flexural torsional buckling of thin-walled columns with open cross-sections are derived from the total potential energy functional by the application of the Euler Lagrange conditions for the extremization (minimization) of the total potential energy functional.

(iv) The governing equations of flexural - torsional buckling of thin-walled columns with open crosssections are a system of three coupled differential equations in terms of the three unknown displacements, namely $u(z), v(z)$ and $\theta(z)$ where $u(z)$ and $v(z)$ are translational displacements, and $\theta(z)$ is the rotational displacement function.

(v) For thin-walled columns with doubly-symmetric open cross-sections, and with hinged (simply supported) conditions at the ends $z=0$, and $z=l$, the system of governing differential equations are uncoupled. The buckling modes and buckling loads are similarly uncoupled.

(vi) For thin-walled columns with open cross-sections that are singly-symmetric (mono-symmetric), and the axial compressive load is applied through the centroid, the buckling behaviour is described by a system of three homogeneous differential equations, two of which are uncoupled. If the thin-walled column in this case is hinged at both ends $z=0$ and $z$ $=l$, the solution of the one uncoupled buckling equation gives the expression for the critical buckling load in the direction of the axis of symmetry.

(vii) For thin-walled columns with open cross-sections that are doubly-symmetric or point symmetric, the buckling equations become uncoupled. The buckling modes are flexural buckling about the $x$ axis, flexural buckling about the $y$ axis of the cross-sections and torsional buckling. Thin-walled columns with doubly-symmetric open cross-sections are thus not subject to flexural - torsional buckling.

\section{REFERENCES}

[1] Zhu S. (2009). elastic flexural - torsional buckling analysis of doubly symmetrical web tapered beams. MSc Thesis, University of Pittsburgh 208.
[2] Huang LH, Li B, Wang YF. (2016). Computational analysis of buckling laods of thin-walled members with open sections. Mathematical Problems in Engineering. http://dx.doi.org/10.1155/2016/8320469

[3] Glauz RS. (2017). Flexural - torsional buckling of general cold-formed steel columns with unequal unbraced lengths. Proceedings of the Annual Stability Conference Structural Stability Research Council San Antonio, Texas 1-18.

[4] ESDEP WG 6 Applied Stability Lecture 6.6.2. Buckling of Real Structural Elements II. http://fgg.web.fgg.uni_Ij.si/ /pmoze/esdep/master/wg06 /10620.htm.

[5] Bhoi R, Kalurkar LG. (2014). Study of buckling behaviour of beam and column subjected to axial loading for various rolled I sections. International Journal of Innovative Research in Science, Engineering and Technology (IJIRSET 3(11): 17639-17645. https://doi.org/10.15680/IJIRSET/ 2014.03.11.077

[6] Howlett JH. (1972). An investigation into the structural behaviour of thin-walled aluminium alloy welded battened struts. MSc Thesis, University of Durham. Durham E - $\quad$ Thesis online https//ethesis.dur.ac.uk/10293, accessed on Jul. 1972.

[7] Trahair NS. (2016). Torsion Equations for lateral buckling. Research Report R 964 July 2016 School of Engineering, The University of Sydney.

[8] Alsayed SH. (1987). Inelastic behaviour of single angle columns. PhD Thesis. The University of Arizona. University Microfilms International. http//ndl.handler.net/10150/184041.1987

[9] Timoshenko SP, Gere JM. (1961). Theory of Elastic Stability. McGraw Hill Kogakusha Ltd, New York.

[10] Chajes A. (1974). Principles of Structural Stability. Prentice Hall, New Jersey.

[11] Wang CM, Wang CY, Reddy JN. (2005). exact solution for buckling of structural members. CRC Series in Computational Mechanics and Applied Mechanics. CRC Press USA.

[12] Allen HG, Bulson PS. (1980). Background to Bulkling McGraw Hill Book Co..

[13] Torsion in Structural Design. http//people.virginia.edu/ttb/torsion.pdf.

[14] Ike CC, Nwoji CU, Ikwueze EU, Ofondu IO. (2017). Solution of the generalised elastic column buckling problem by the galerkin variational method. International Journal of Research in Applied Science and Engineering Technology (IJRASET) 5(1): 468-475.

[15] Mama BO, Ike CC, Nwoji CU, Onah HN. (2017). Application of the finite fourier sine transform method for the flexural - torsional buckling analysis of thinwalled columns. IOSR Journal of Mechanical and Civil Engineering (IOSRJMCE) 14(2): 51-60. https://doi.org/10.9790/1684-1402015160

[16] Onah HN, Ike CC, Nwoji CU. (2017). Flexural torsional buckling analysis of thin-walled columns using the fourier series method. International Journal of Advanced Engineering Research and Science (IJAERS) 4(3): 292-298. https//dx.doi.org/10.22161/ijaers.4.3.45

[17] Det NV. (2004). Buckling Strength Analysis of Bars and Frames and Spherical Shells Classification Notes No 3, 1 Norway April.

[18] Trahair NS. (1993). Flexural - Torsional Buckling of Structures. CRC Press Tokyo. 